\author{
Military Technical College \\ Kobry El-Kobbah, \\ Cairo, Egypt.
}

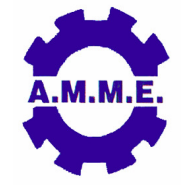

\title{
COMPARISON OF AERODYNAMIC FORCES USING A
COMMENDED AND A NON-RECOMMENDED LIFT SPOILER \\ COMPARISON OF AERODYNAMIC FORCES USING A
RECOMMENDED AND A NON-RECOMMENDED LIFT SPOILER ON A PASSENGER CAR
}

\author{
$15^{\text {th }}$ International Conference \\ on Applied Mechanics and \\ Mechanical Engineering.
}

\author{
M. G. Yazdani", P. G. O. Sumajaya ${ }^{* *}$ and M. N. Arifin ${ }^{* *}$
}

\begin{abstract}
An attempt was made to compare the aerodynamic forces acting on Subaru WRX STi version IV (SPC) with a recommended World Rally Championship (WRC) and a non-recommended GT Wing Type-A (GTA) spoilers. The experiment was done with the following combination, SPC, SPC+WRC (REC), and SPC+GTA (NRE). The above experiments were carried out at a Reynolds Number (R) range of $2.19 \times 10^{4}$ to $10.94 \times 10^{4}$ and an angle of attack $(\alpha)$ of $0^{\circ}$ to $20^{\circ}$. The lift coefficient $\left(C_{L}\right)$, drag coefficient $\left(C_{D}\right)$ and side force coefficient $\left(C_{S}\right)$ are computed from the measured data From the analyzed data, it was found that, for REC. $C_{L}$ decreased in the almost all the investigated ranges of $\alpha$ and $R$. The maximum decrease in $C_{L}$ is about $40 \%$ (at $\alpha$ $=20^{\circ}$. . On the other hand for NRE, $C_{L}$ increases for almost all the investigated ranges of $\alpha$ and $R$. The maximum increase of $C_{L}$ is about $177 \%$. At, $2.19 \times$ $10^{4} \leq R \leq 6.57 \times 10^{4}$, there is little or no changes in the value of $C_{D}$ for REC and SPC in the investigated ranges of $\alpha$. It was found that value of $C_{S}$ for REC is less than that of NRE in the investigated ranges of $\alpha$ and $R$.
\end{abstract}

\section{KEY WORDS}

Aerodynamics, passenger car, lift spoiler, angle of attack, Reynolds's number.

\footnotetext{
Senior Lecturer, Dept. of Mechanical Engineering, Institute Technology Brunei-University.

${ }^{\star *}$ Student, Dept. of Mechanical Engineering, Institute Technology Brunei-University.
} 


\section{NOMENCLATURE}

$\begin{array}{ll}D & \text { Drag force } \\ S & \text { Side force } \\ L & \text { Lift force } \\ u & \text { Velocity of air in wind tunnel } \\ C_{D} & \text { Drag coefficient } \\ C_{S} & \text { Side coefficient } \\ C_{L} & \text { Lift coefficient } \\ A_{D} & \text { Fontal projected area } \\ A_{S} & \text { Side projected area } \\ A_{L} & \text { Plan area } \\ \rho & \text { Density of air }\end{array}$

\section{INTRODUCTION}

\section{Background}

The performance and handling of automobile are significantly affected by its aerodynamic properties. One of the main causes of aerodynamic is about lifting force. This will influence all the aspect of the vehicles such as overall performance, fuel consumption, safety and stability. The addition of rear spoiler to an aerodynamically optimized car body, leads to decrease lift coefficients. In an aerodynamic field, the main important thing to get the stability and performance is to design a vehicle with low lift coefficient. The reduction of lift and flow separation is the key results that will be a point of discussion. Rear spoiler will reduce the flow separation at the trunk that causing the turbulent airflow.

Since the above is both shape and flow dependant, no complete theory is available. Experimental techniques are thought to be the best to look into the aerodynamics of such a problem. Wind tunnels and flumes are commonly used for controlled experimental studies to understand the physics of a particular flow field or to obtain data necessary for designing or improving a given product. Less common, but by no means less useful, are facilities in which an object can be towed or propelled through air or water. [1]

\section{Literature Survey}

Ways of improving the aerodynamic characteristics of large vehicles such as trucks and buses are being actively promoted. The fact that aerodynamic is effective at lowering fuel consumption has finally gotten through to people who use trucks, making the aerodynamic characteristics of present-day trucks a key selling point. For the owners of fleets of trucks, buses, horse carriers and other vehicles which are towed, fuel consumption is a pressing problem. The drag coefficient $\left(C_{D}\right)$ of most trucks on the road today ranged between 0.7 and 0.9 [2]. 
Drag coefficients with load $\left(\mathrm{C}_{D}\right.$ and $\left.\mathrm{C}_{\mathrm{DA}}\right)$ differ considerably according to whether or not a truck is carrying a load or is equipped with a wind deflector. When cargo is piled up higher than the height of the truck's cabin, both $C_{D}$ and $C_{D A}$ increase, but can be decreased with the addition of a wind deflector; and when the height of the cargo is less than that of the cabin and a wind deflector is used, then both $C_{D}$ and $C_{D A}$ increase drastically]. In the present studies a simple flat-plate deflector was attached to the roof of a model car and inclined at $0^{\circ}-5^{\circ}$.

Hands. S.J and. Zdravkovich, M.H [3] tested two types of car: a standard coupé and an estate version towing a caravan. A deflector was used to reduce the drag for both the above combination. A smoke-visualisation technique was utilised to determine the optimal angle for the deflector using a small smoke wind-tunnel. Drag and lift forces were measured for the two car-caravan combinations in a larger wind tunnel. It was found that the best position for the deflector was along the trailing edge of the roof (for either the coupé or the estate car). Some additional reduction in drag was achieved by fixing a horizontal plate along the towing bar.

Koike et. al, [4]_ tested bump-shaped vortex generators at the roof end of a sedan to reduce drag. Their paper presents the optimization result, the effect of vortex generators in the flow field and the mechanism by which these effects take place.

The behavior of different add-on parts to a basic car model has been studied experimentally [5]. Experiments were conducted with different add-on parts, like rear end spoiler and front end spoiler in order to find out how these add-on parts influence the drag and lift coefficients of a basic car model. The experiment was done in Jadavpur University low turbulence subsonic closed circuit wind tunnel at different Reynolds number The results from the experiment indicated that the addition of different add-on parts like front end spoiler and rear end spoiler to a basic car model reduces the lift coefficient to a considerable amount while the drag coefficient is reduced by a small amount. It was concluded that the addition of these add-on parts like rear-end spoiler etc increases the aerodynamic stability of a basic car model and hence they can be used in real life which will be an added advantage.

An assessment of the role of fluid dynamic resistance and aerodynamic drag and the relationship to energy use in the United States is presented [6]. Existing data indicates that $16 \%$ of the total energy consumed in the United States is used to overcome aerodynamic drag in transportation systems. Application of existing pressure drag reduction technologies to all ground vehicles within the United States will reduce yearly energy costs by 20 billion dollars.

Three simple, low cost aerodynamic drag reduction devices have been developed for application to the trailer of a tractor-trailer truck [7]. The three devices have undergone extensive operational testing where they have amassed over 85,000 miles of use. These technologies have shown a combined fuel savings of $10 \%$ at an average speed of $47.5 \mathrm{mph}$. This improvement in fuel economy correlates to an equivalent drag reduction of approximately $30 \%$ with a corresponding drag coefficient of 0.45 . Observations and anecdotal evidence from the test activity have shown that the addition of these devices to the trailers has not had a negative impact on either the operational utility of the trailers or the maintenance procedures and requirements 
A numerical study [8] with two different types of simulations was made, one for the flow around a simplified high speed passenger ear with a rear-spoiler and the other for the flow without a rear-spoiler. The standard k-s model was selected to numerically simulate the external flow field of the simplified Camry model with or without a rear-spoiler. Through an analysis of the simulation results, a new rear spoiler was designed and it shows a mild reduction of the vehicle aerodynamics drag. This leads to less vehicle fuel consumption on the road.

\section{METHODOLOGY}

\section{Passenger Car}

In this experiment, the model that is selected was Subaru WRX STi Version IV which is one of the favourite saloon passenger cars in Brunei. For testing in a wind tunnel a model with a scale of 1: 25 is used. This model was bought form model shop as shown in Fig. 1. It was tested for the effect on the aerodynamics forces both with the spoiler and without spoiler.

\section{Models}

\section{Without spoilers}

A scale of 1:25 model, which is available in the market, is used in the present investigation based on a blockage age ratio of less than $5 \%$. The dimensions of the model are shown in Fig. 2.

\section{WRC spoiler}

The WRC spoiler (Fig. 3) (World Rally Championship) is selected since it is dedicated for Subaru rally car and furthermore it is famous among Subaru car user in Brunei. The spoiler model was bought in the model shop.

\section{GT wing type-a spoiler}

The Grand Touring or known as GT wing spoiler (Fig. 4) has been scaled down from the actual size. It was designed using Perspex.

\section{End Plate}

An end plate was used to find the lift coefficient. Based on the width (W) and length $(\mathrm{L})$ the end plate dimension is $2 \mathrm{~W} \times 1.5 \mathrm{~L}$. The end plate is shown in Fig. 5.

\section{Wind Tunnel}

All the experiments were carried out in a subsonic wind tunnel (Fig. 6) (Model HM 170 , Gunt) in the Institute of Technology. The subsonic wind tunnel has a square measurement working section $(292 \mathrm{~mm} \times 292 \mathrm{~mm})$. It has a total length of $450 \mathrm{~mm}$. The air is drawn from the atmosphere via the streamlined funnel. Since it has a smaller working section, as such to minimize the effect of side walls a blockage ratio of $5 \%$ is chosen for the model to be tested in the wind tunnel. It is known from 
experiments that for a blockage ratio of $\leq 5 \%$, the side walls have no or little effect on the models.

Any transverse flow components are filtered out in the flow straighter. It is made of a tubular honeycomb structure. The air exits from the flow straighter as a parallel flow and is accelerated to roughly 3.3 times its original velocity in the jet. The flow forces at the models are measured by an electronic two-component force measuring device. This device consists of a force transducer and a measurement amplifier with display. With the above arrangement the irregularity of velocity is within $\pm 2 \%$.

For measuring drag and side forces the models are placed horizontally (Fig. 7) and for lift measurements it is placed transversely with the vertical using an end plate (Fig. 8)

The experiments were carried out at $0^{\circ} \leq \alpha \leq 20^{\circ}$ and $5 \leq$ free stream velocity $(u) \leq 15$ $\mathrm{m} / \mathrm{s}$. This is equivalent to a speed of 18 to $54 \mathrm{~km} / \mathrm{hr}$. But due to the limitation on the wind tunnel, the authors were satisfied with the above range of $u$. The authors believe that as all the experiments are done in turbulent $R$, the comparison between SPC, NRE, and REC will be still valid. Based on the width, the $R$ was in the range of $1.77 \times 10^{4}$ to $7.8 \times 104$. The $\alpha$ and direction of positive quantities is shown in Fig. 9 .

\section{Drag, Lift and Side Force Coefficient}

$C_{D}, C_{L}$ and $C_{S}$ for SPC, REC and NRE were computed from wind tunnel experiment for $1.49 \times 104 \leq R \leq 7.23 \times 10^{4}$ and $0^{\circ} \leq \alpha \leq 30^{\circ}$. The following equations were used for computing the above coefficients:

$$
\begin{aligned}
& C_{D}=\frac{D}{\frac{1}{2} \rho u^{2} A_{D}} \\
& C_{S}=\frac{S}{\frac{1}{2} \rho u^{2} A_{S}} \\
& C_{L}=\frac{L}{\frac{1}{2} \rho u^{2} A_{L}}
\end{aligned}
$$

\section{Correction of Force Data for Lever Effect}

The force $F_{A}$ (Lift) and $F_{W}$ (Drag) are converted by means of the lever arm $a$ of the model holder into proportional moments M, (Fig. 10.) which deform a bending and torsion beam. The deformation is measured with a strain gauge and displayed digitally on the two-channel amplifier as force. Lever arms other than $a=310 \mathrm{~mm}$ involve correction of the display force $\mathrm{F} ; F_{\text {corr. }}=F .310 / \mathrm{a}$ with a in $\mathrm{mm}$. 


\section{RESULTS AND DISCUSSIONS}

The objective of this present paper is to compare the results between an SPC, REC and NRE in terms of the aerodynamic coefficient and draw some conclusions, based on experiments. The above is discussed in detail in the following sub-sections.

\section{Lift Force Coefficient}

The $C_{L}$ versus a for SPC, REC and NRE the investigated ranges of $R$ are shown in Figs 11 to 15 , respectively.

Though, the data for this particular combination is difficult to find, but for the sake of comparison the authors have used the published data [8] for Proton Saga BLM car without and with aerofoil spoiler in the range of $70 \mathrm{~km} / \mathrm{h}$ to $110 \mathrm{~km} / \mathrm{h}$. It was found that the range of $C_{L}$ is 0.118 to 0.115 and 0.112 to 0.115 without and with the above mentioned spoiler respectively. The above data is well compared with present investigation. It is also observed from the above data that there is little changes in the value of $C_{L}$ in the above mentioned speed range, which confirms the assumption mentioned previously.

From the above figures it is seen that in the investigated ranges of $R$ and $\alpha$ the $C_{L}$ is always higher for NRE. Though the GTA is used for decreasing lift, but in this particular case it is increasing the lift. It can be explained as follows. The lift is reduced by deflecting the flow, which in turn increase the $C_{D}$. But due to different height of GTA and height of rear of SPC, it might be possible that some of the the flow is allowed to flow in instead of deflecting and thereby increase the lift instead of decreasing it. The above explanation is suported by the $C_{D}$ values at $\alpha=0^{\circ}$. This might also need to be be cofirmed with a flow visualization.

For $\mathrm{R}=2.19 \times 10^{4}, \mathrm{C}_{\mathrm{L}}(\mathrm{REC})=\mathrm{C}_{\mathrm{L}}(\mathrm{SPC})=0.25$ at $\alpha=0^{\circ}, \mathrm{C}_{\mathrm{L}}(\mathrm{REC})>\mathrm{C}_{\mathrm{L}}(\mathrm{SPC})$ at $5^{\circ} \leq \alpha \leq 10^{\circ}$ and $C_{L}(R E C)<C_{L}(S P C)$ at $\alpha=20^{\circ}$.

For $\mathrm{R}=4.38 \times 10^{4}, \mathrm{C}_{\mathrm{L}}(\mathrm{REC}) \approx \mathrm{C}_{\mathrm{L}}(\mathrm{SPC})$ at $0^{\circ} \leq \mathrm{\alpha} \leq 15^{\circ}$ and $\mathrm{C}_{\mathrm{L}}(\mathrm{REC})<\mathrm{C}_{\mathrm{L}}(\mathrm{SPC})$ at $\alpha$ $=20^{\circ}$.

For $6.57 \times 10^{4} \leq R \leq 10.95 \times 10^{4}, C_{L}(R E C) \leq C_{L}(S P C)$ in the investigated ranges of $\alpha$.

It may be also observed that, at $\alpha=0^{\circ}$ and $R=4.38 \times 10^{4}, C_{L}$ (NRE) is high (Fig. 11) compared to other ranges of $\mathrm{R}$. The authors have no explanation about the above at present. Also the curve trend for $0^{\circ} \leq \alpha \leq 5^{\circ}$ and $15^{\circ} \leq \alpha \leq 20^{\circ}$ is different. The authors believe that it may be due to the effect of shape. All the above needs to be investigated further with flow visualization.

In general, $C_{L}(R E C)<C_{L}(S P C)<C_{L}(N R E)$ for most of the ranges of $R$ and $\alpha$. It might be said that REC has an advantage over NRE for this model of SPC with respect to $\mathrm{C}$. 


\section{Drag Force Coefficient}

The $C_{D}$ versus $\alpha$ for SPC, REC and NRE the investigated ranges of $R$ are shown in Figs. 16 to 20 , respectively. From the above curves, it is observed that there are little or no differences in the values of $C_{D}$ for the three cases (SPC, REC and NRE). The above might be explained as follows. It is reported [10] that $70 \%$ of drag is due to front design, $20 \%$ and $10 \%$ is due to rear design and body friction respectively. From the above it is noted that the changes in rear design is responsible for $20 \%$ changes in $C_{D}$. Since adding a spoiler may attribute to a minor change of rear design. As such the changes in $C_{D}$ are not significant for the three cases. It might be said that there is no significant difference between using REC and NRE, if one consider the $C_{D}$.

\section{Side Force Coefficient}

The $\mathrm{C}_{S}$ versus $\alpha$ for SPC, REC and NRE in the investigated ranges of $\mathrm{R}$ is shown in Figs. 21 to 25, respectively. From the above figures (Figs. 21. to 25), it is seen that $\mathrm{C}_{s}$ increases with increase of $\alpha$ for all of the investigated ranges of $\mathrm{R}$. There are little or no changes in the value of $C_{s}$ for the three cases for $\alpha \leq 15^{\circ}$. For $\alpha=20^{\circ}, C_{s}$ $(R E C)<C_{S}(S P C)<C_{S}(N R E)$, in the investigated ranges of $R$. As such it might be said that if there is presence of significant side wind (Velocity of side wind ( $\mathrm{V}_{\mathrm{CSW}} \geq$ $0.35 \times$ Car velocity $\left(\mathrm{V}_{\mathrm{SPC}}\right)$ the NRE will be subjected to more $\mathrm{C}_{\mathrm{S}}$ compared to REC. As such the NRE might be susceptible to toppling by the side wind for the above condition.

\section{CONCLUSIONS}

From the above discussions, the following conclusions can be drawn:

1. In general, $C_{L}(R E C)<C_{L}(S P C)<C_{L}(N R E)$ for most of the ranges of $R$ and $\alpha$. It might be said that REC has an advantage over NRE for this model of SPC with respect to $\mathrm{C}_{\mathrm{L}}$.

2. The changes in $C_{D}$ are not significant for the three cases. It might be said that there is no significant difference between using REC and NRE, if one consider the $C_{D}$.

3. It might be said that if $\mathrm{V}_{\mathrm{CSW}} \geq 0.35 \mathrm{~V}_{\mathrm{SPC}}$ the NRE combination might be susceptible to toppling due to $\mathrm{C}_{\mathrm{s}}$.

4. From the above, it might be concluded that, as it is evident from experiment that a non-recommended spoiler can increase the lift instead decreasing as such if anyone need to install a spoiler for a passenger car which is not recommended by the car manufacturer then it is to be tested in a wind tunnel.

5. Further work is suggested to test a series of different combination of recommended and non-recommended lift spoilers. It also recommends doing a flow visualization, and to look into the physics of flow.

\section{REFERENCES}

[1] Gad-el-Hak M., "The Water Towing Tank as an Experimental Facility", Experiments in Fluids, Springer Verlag , Berlin, pp. 289 - 297. (1987), 
[2] Muto S., "Automobile Aerodynamics", Osami Suzuki, Tokyo,(1986).

[3] Hands S. J. and Zdravkovich M. H., "Drag reduction for a passenger car towing a caravan", $4^{\text {th }}$ Colloquium on Industrial Aerodynamics (Vehicle Aerodynamics), Aachen, June 18,(1960).

[4] Koike M., Nagayoshi T. and Hamamoto N., "Research on aerodynamic drag reduction by vortex Generators", Technical Review, Mitsubishi Motors, No.16, , pp 11-16, (2004).

[5] Mitra D., "Effect of Relative Wind on Notch Back Car with Add-On Parts", Int. J. of Eng. Sci. and Tech., Vol. 2(4), pp.472-476, (2010)

[6] Wood R. M., "Impact of Advanced Aerodynamic Technology on Transportation Energy Consumption", SAE 2004-01-1306, (Nov. 2004)

[7] Wood R. M. and Bauer S. X. S., "Simple and Low-Cost Aerodynamic Drag Reduction Devices for Tractor-Trailer Trucks", SAE 2003-01-3377, Nov. 2003

[8] $\mathrm{Hu}$ X. and Wong T.T. , "A Numerical Study On Rear-spoiler Of Passenger Vehicle", World Academy of Science, Engineering and Technology 81, pp. 636641, (2011)

[9] Ridhwan C.Z., "Aerodynamics of Aftermarket Rear Spoiler", Report is submitted in partial fulfillment of the requirements for the award of the degree of Bachelor of Mechanical Engineering with Automotive Engineering, Faculty of Mechanical Engineering, University Malaysia Pahang, (Nov. 2008).

[10] Morel T. and Dalton C., "Aerodynamics of Transportation", the American Society of Mechanical Engineers, New York, (1979).

\section{FIGURES:}

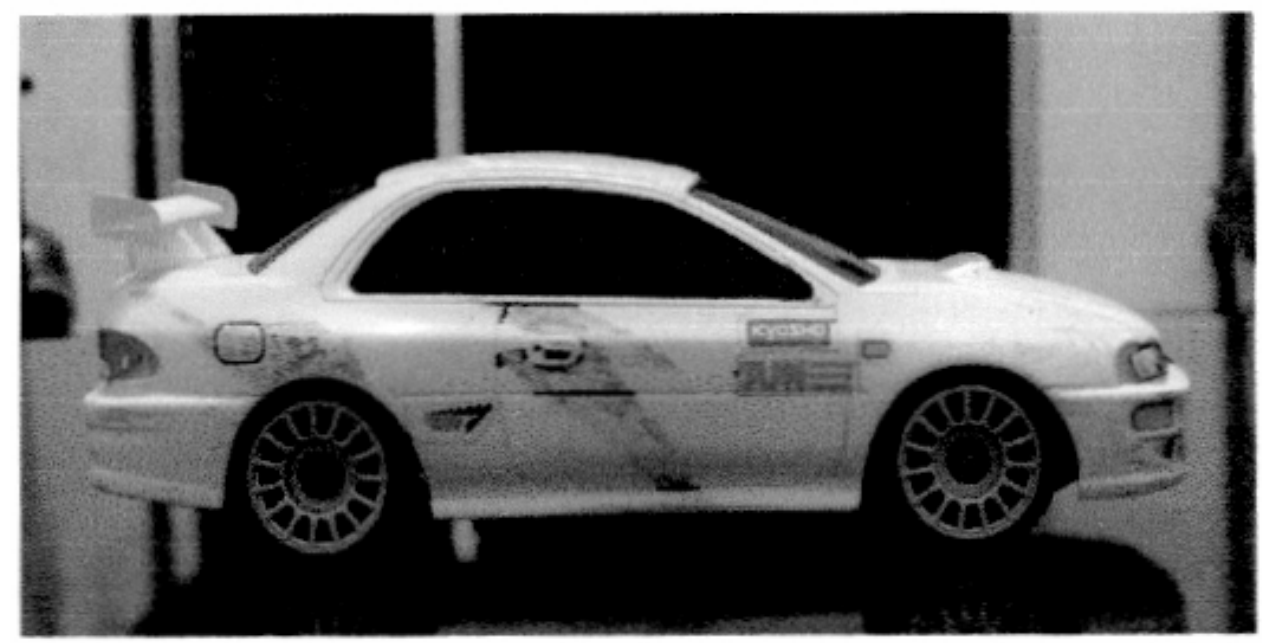

Fig. 1. Picture of the passenger car in the present investigation. 

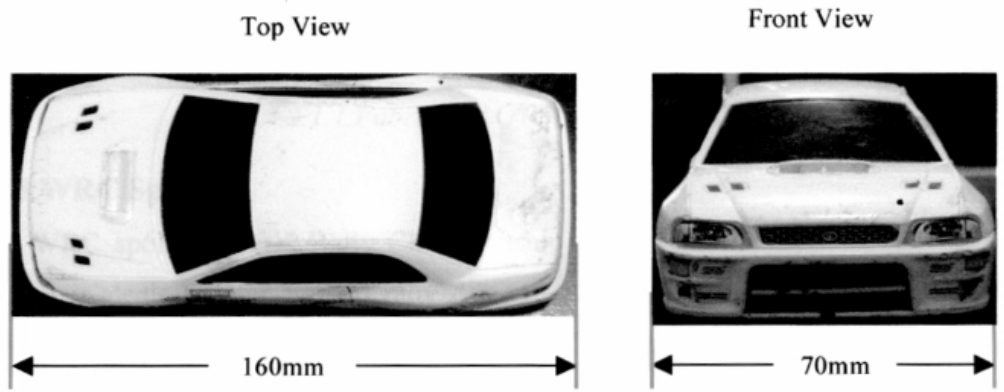

Side View

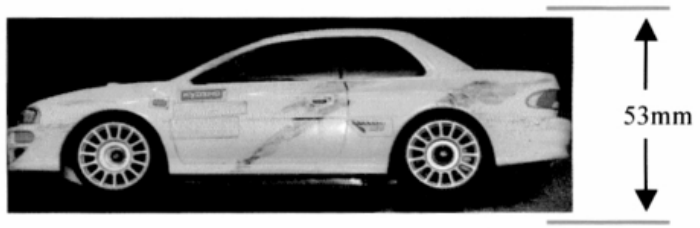

Fig. 2. Dimensions of the model in the present investigation.

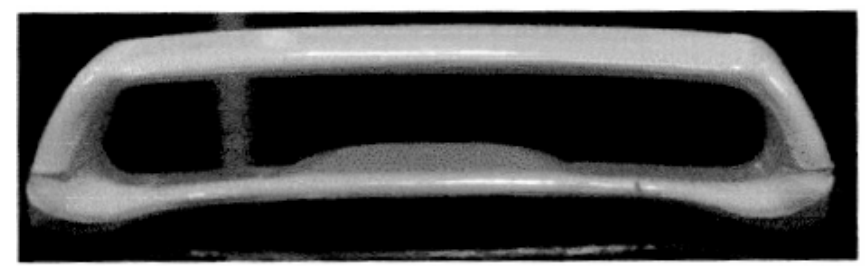

Fig. 3. WRC Spoiler.

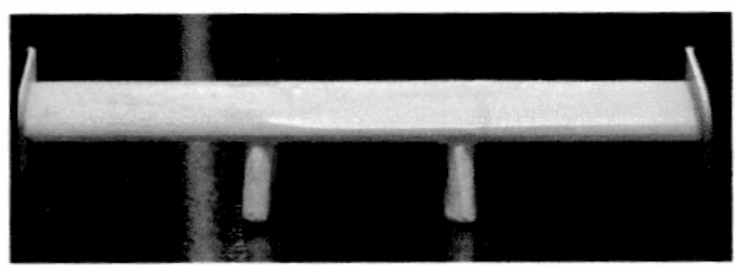

Fig. 4. GT Wing Type-A Spoiler.

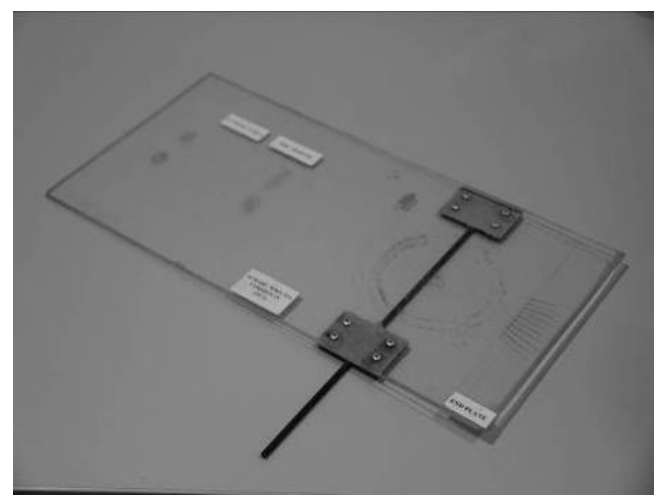

Fig. 5. End Plate.

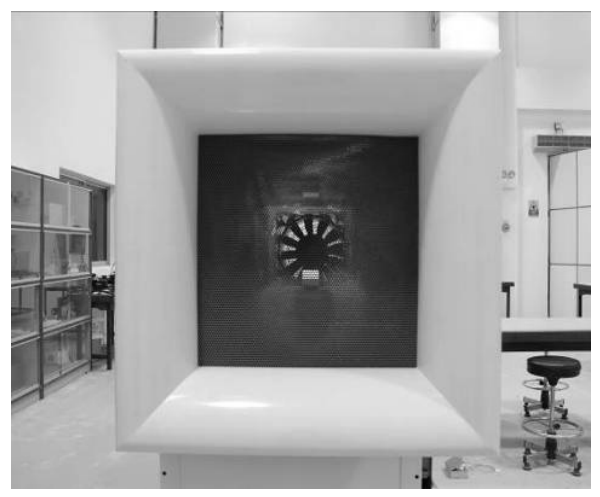

Fig. 6. Tubular honeycomb structure in the flow straighter. 


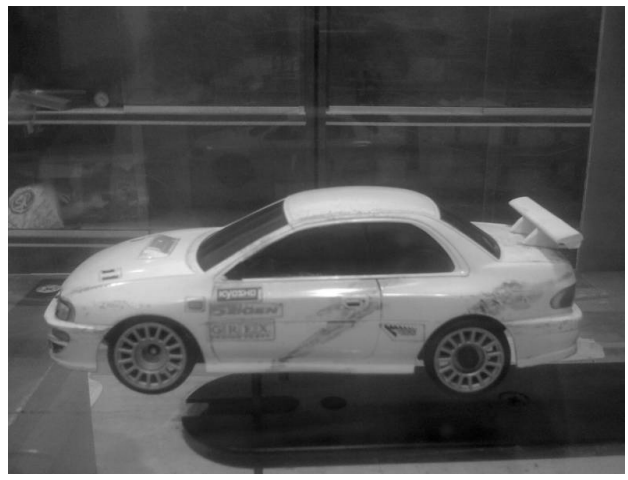

Fig. 7. Model orientation for drag and side force measurement.

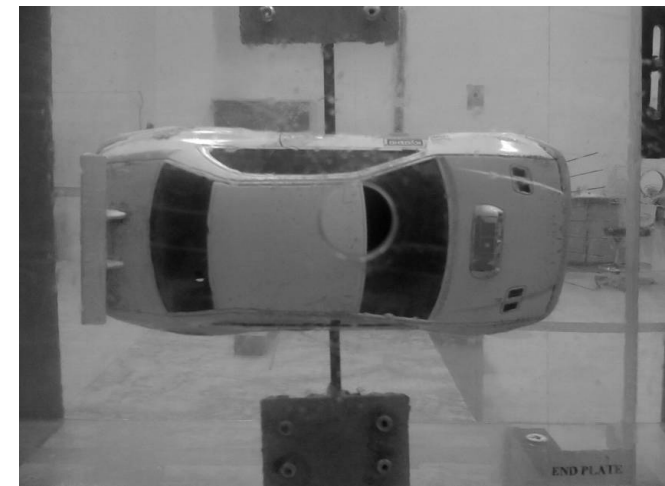

Fig. 8. Model orientation for lift force measurement.

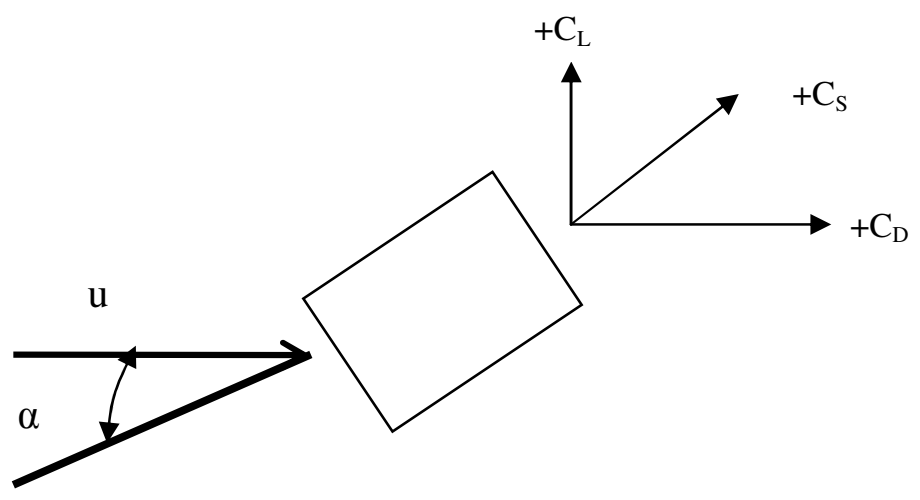

Fig. 9. Angle of attack ( $\alpha$ ) with car longitudinal axis and directions of positive quantities.

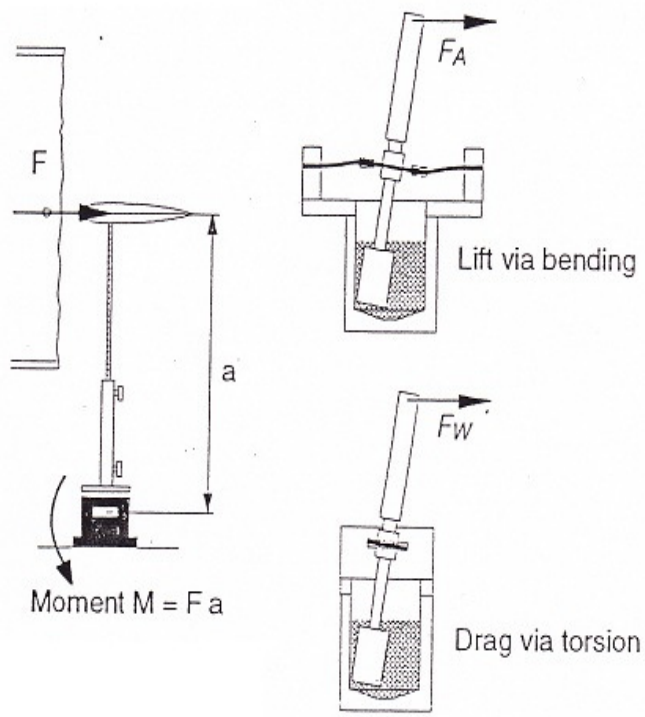

Fig.10. Connection of Model and Force Transducer. 


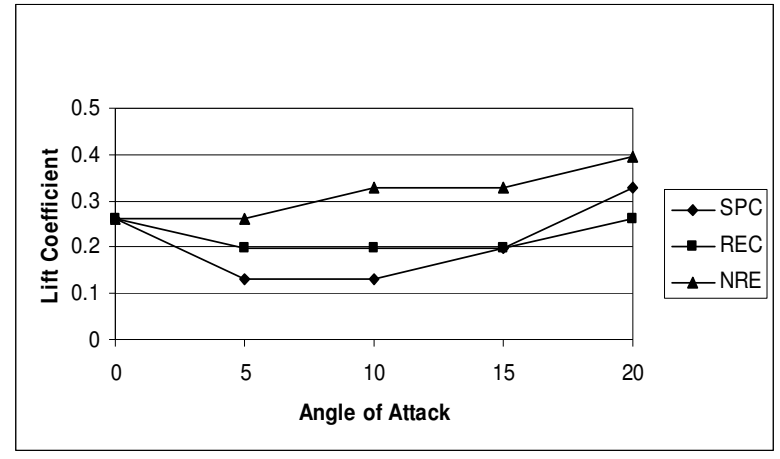

Fig 11. $C_{L}$ Vs. $\alpha, R=2.19 \times 10^{4}$

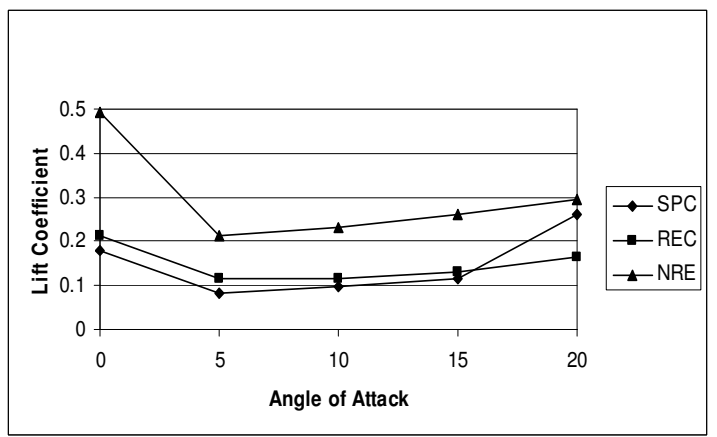

Fig 12. $C_{L}$ Vs. $\alpha, R=4.38 \times 10^{4}$.

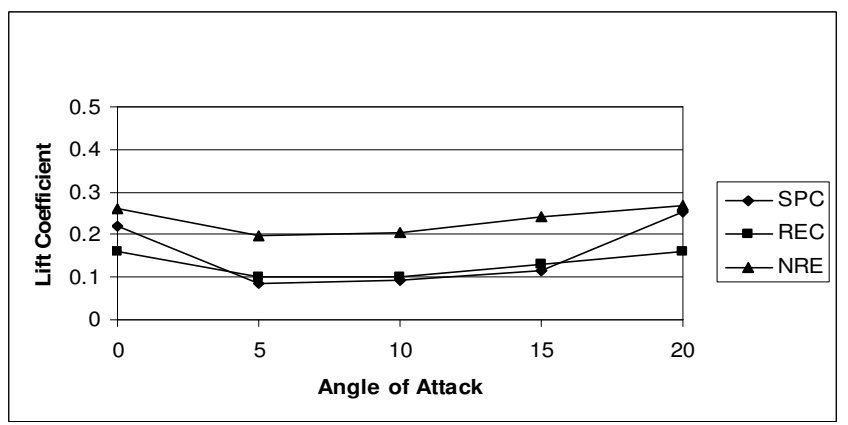

Fig. 13. $C_{L}$ Vs. $\alpha, R=6.57 \times 10^{4}$.

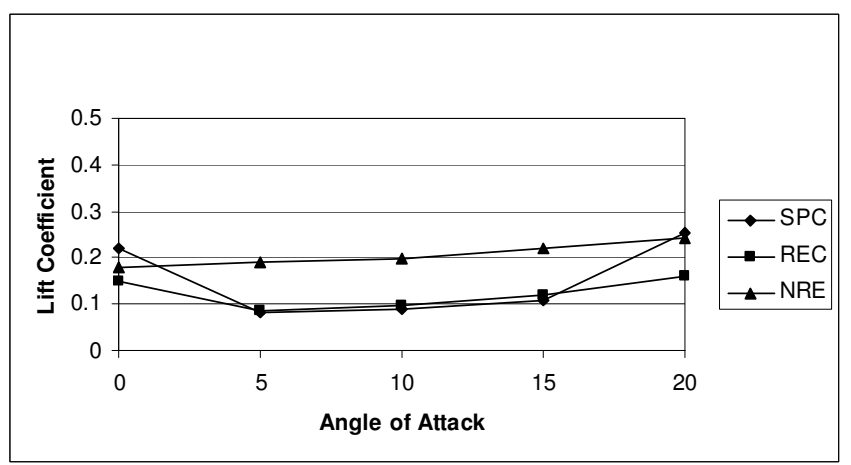

Fig. 14. $C_{L}$ Vs. $\alpha, R=8.76 \times 10^{4}$ 


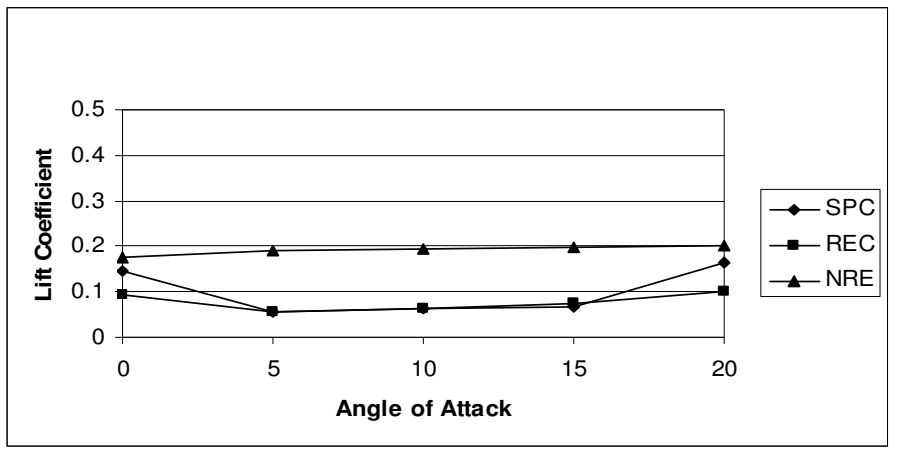

Fig. 15. $C_{L}$ Vs. $\alpha, R=10.95 \times 10^{4}$

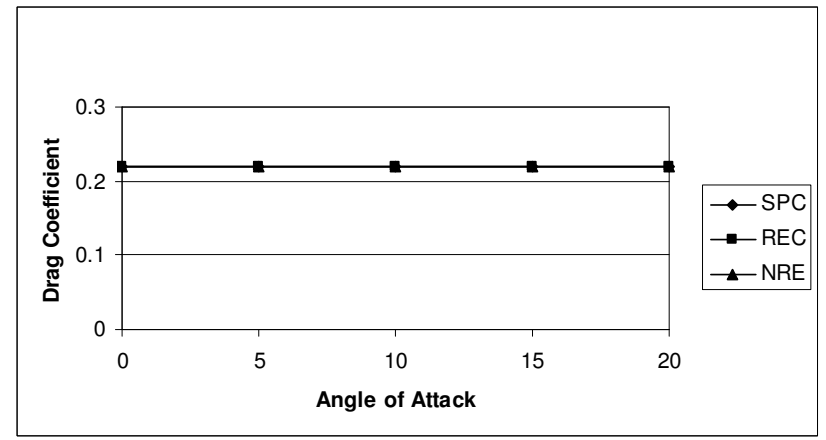

Fig. 16. $C_{D}$ Vs. $\alpha, R=2.19 \times 10^{4}$

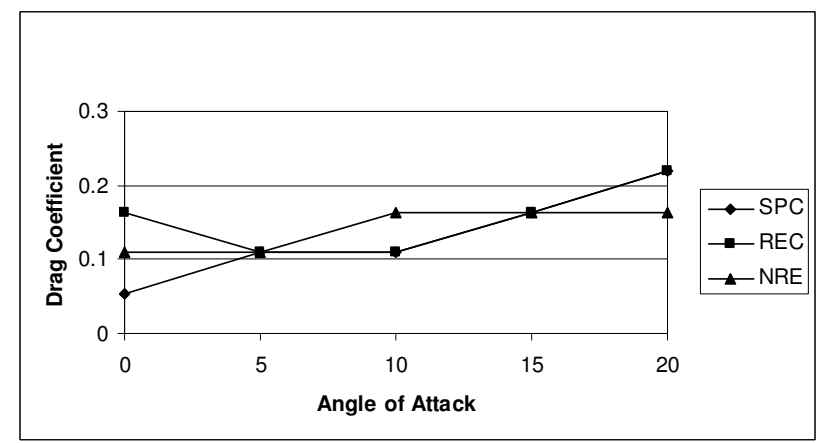

Fig. 17. $C_{D}$ Vs. $\alpha, R=4.38 \times 10^{4}$.

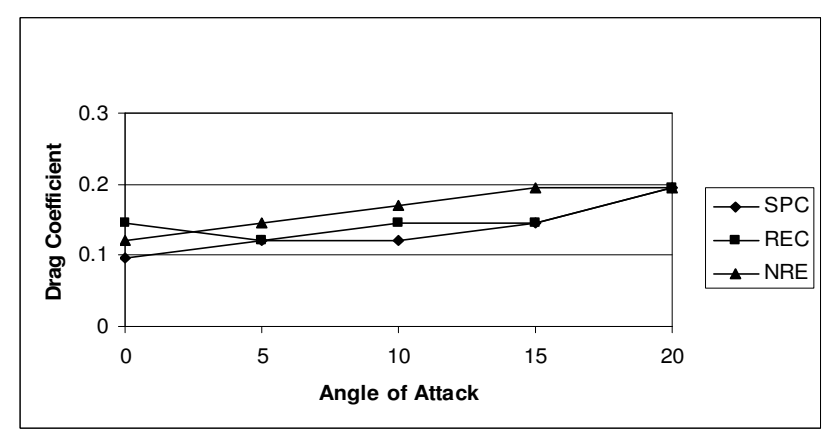

Fig. 18. $C_{D}$ Vs. $\alpha, R=6.57 \times 10^{4}$. 


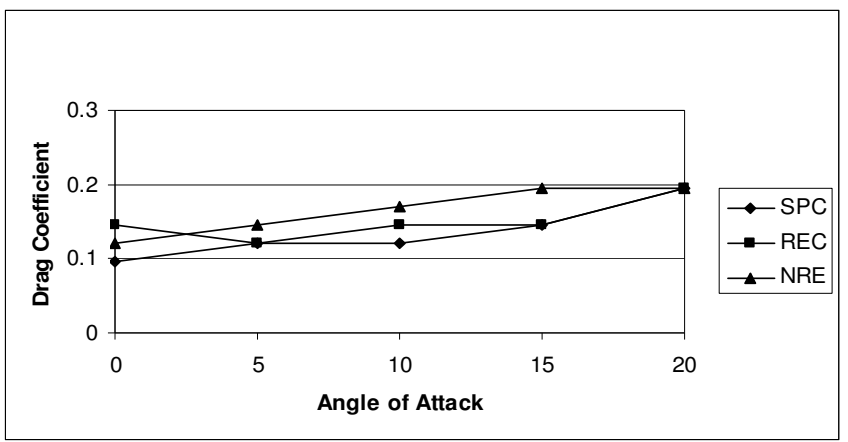

Fig. 19. $C_{D}$ Vs. $\alpha, R=8.76 \times 10^{4}$.

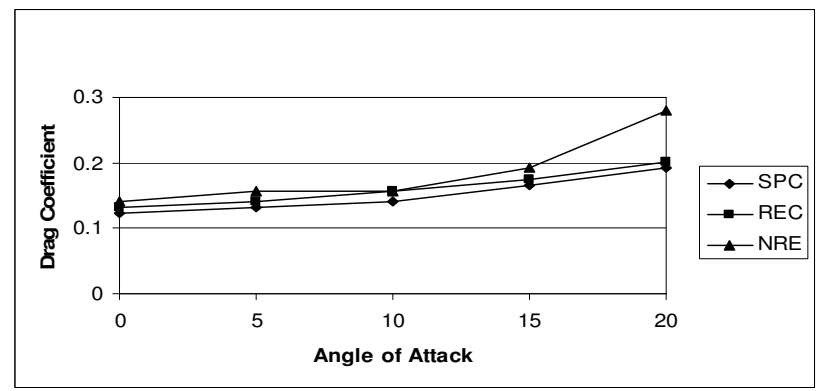

Fig. 20. $C_{D}$ Vs. $\alpha, R=10.95 \times 10^{4}$

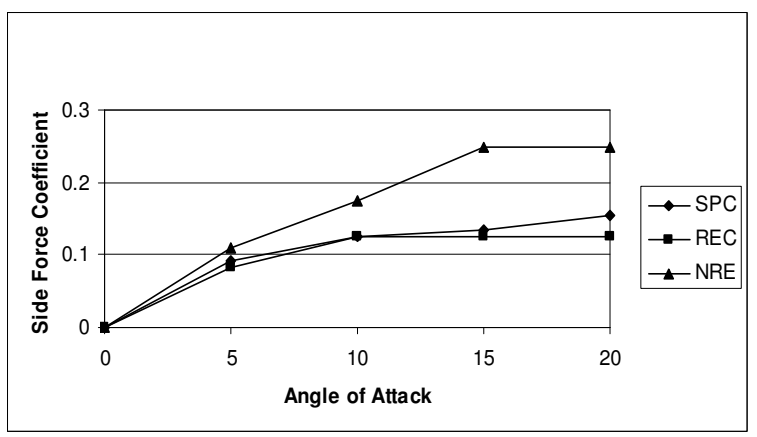

Fig. 21. $C_{s}$ Vs. $\alpha, R=2.19 \times 10^{4}$

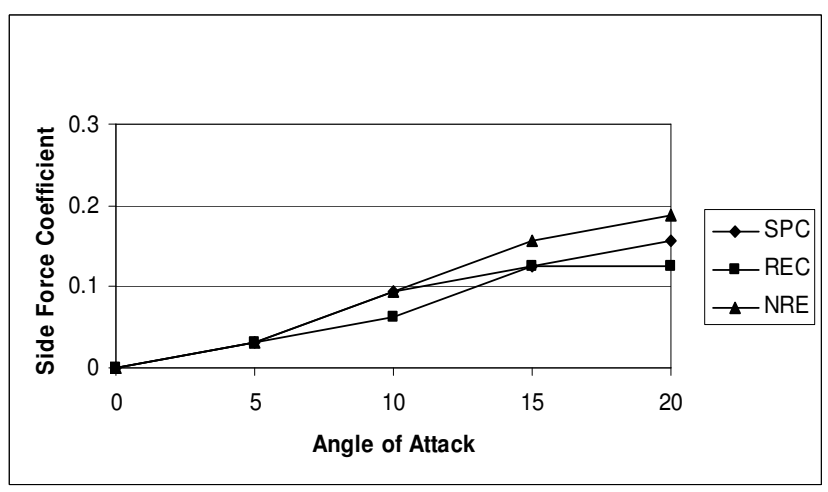

Fig. 22. $C_{s}$ Vs. $\alpha, R=4.38 \times 10^{4}$. 


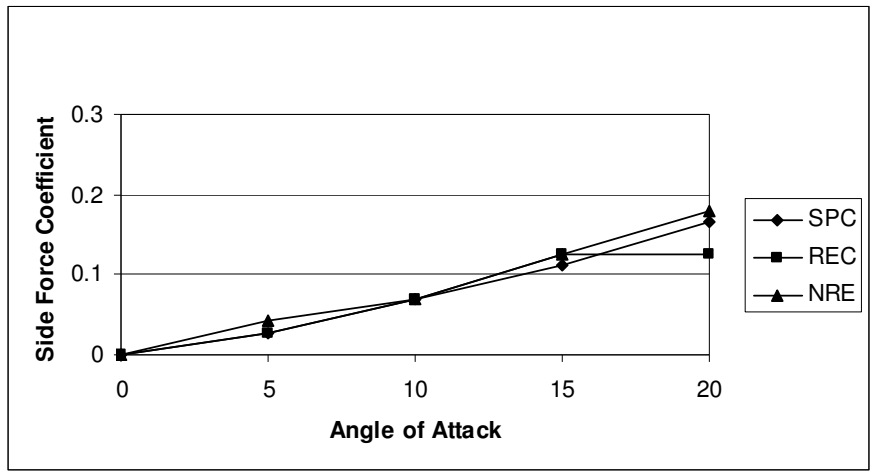

Fig. 23. $C_{s}$ Vs. $\alpha, R=6.57 \times 10^{4}$.

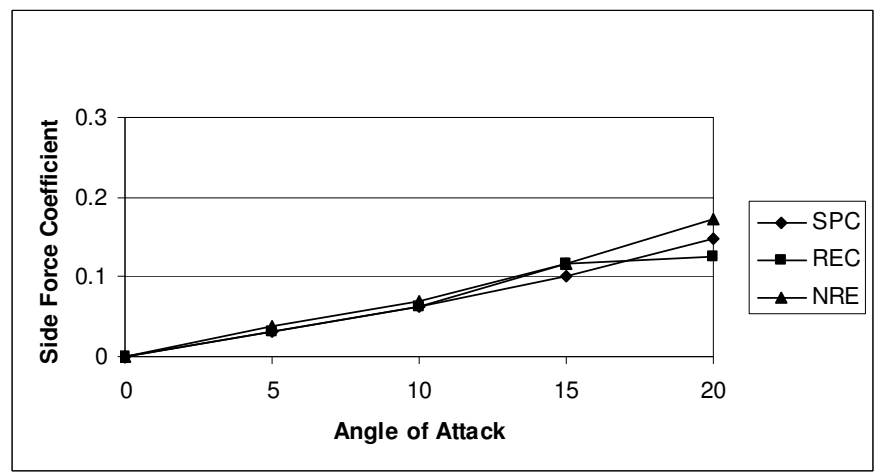

Fig. 24. $C_{s}$ Vs. $\alpha, R=8.76 \times 10^{4}$.

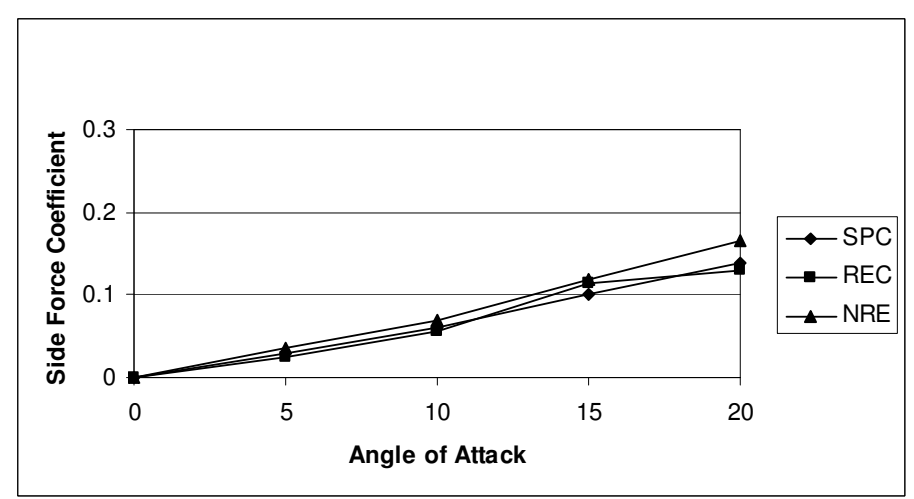

Fig. 25. $C_{s}$ Vs. $\alpha, R=10.95 \times 10^{4}$ 